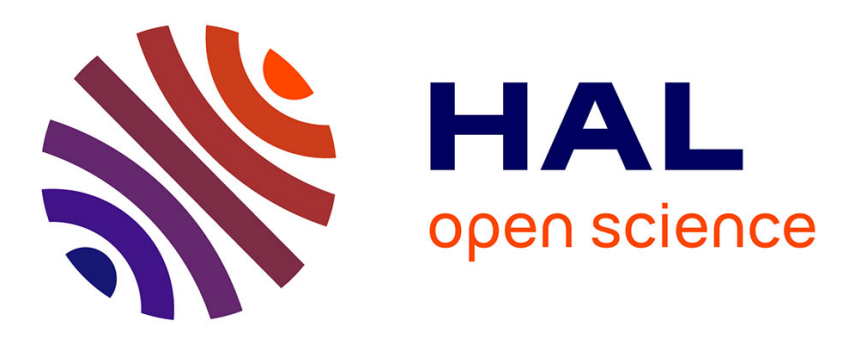

\title{
Statics and dynamics of wetting on thin solids
}

Martin E. R. Shanahan

\section{To cite this version:}

Martin E. R. Shanahan. Statics and dynamics of wetting on thin solids. Revue de Physique Appliquée, 1988, 23 (6), pp.1031-1037. 10.1051/rphysap:019880023060103100 . jpa-00245905

\section{HAL Id: jpa-00245905 https://hal.science/jpa-00245905}

Submitted on 1 Jan 1988

HAL is a multi-disciplinary open access archive for the deposit and dissemination of scientific research documents, whether they are published or not. The documents may come from teaching and research institutions in France or abroad, or from public or private research centers.
L'archive ouverte pluridisciplinaire HAL, est destinée au dépôt et à la diffusion de documents scientifiques de niveau recherche, publiés ou non, émanant des établissements d'enseignement et de recherche français ou étrangers, des laboratoires publics ou privés. 
Classification

Physics Abstracts

68.10

\title{
Statics and dynamics of wetting on thin solids
}

\author{
Martin E. R. Shanahan \\ Ecole Nationale Supérieure de Chimie de Mulhouse, 3 rue Alfred-Werner, 68093 Mulhouse Cedex, France \\ Centre de Recherches sur la Physico-Chimie des Surfaces Solides, 24 Avenue du Président Kennedy, 68200 \\ Mulhouse, France
}

(Reçu le 28 septembre 1987, révisé le 11 décembre 1987, accepté le 18 décembre 1987)

\begin{abstract}
Résumé. - L'état final d'équilibre, ainsi que la dynamique d'étalement vers l'équilibre, ont été étudiés pour le cas d'une goutte posée sur un solide mince. En ce qui concerne l'équilibre, les deux configurations correspondant à la symétrie axiale et à la symétrie cylindrique ont été considérées. La tension superficielle du liquide peut provoquer une courbure importante du solide, initialement plan, surtout dans le deuxième cas. La dynamique d'étalement est accélérée sur un substrat mince. Des contributions à la fois de l'énergie élastique restituée et des effets capillaires compensent la résistance visqueuse à l'écoulement.
\end{abstract}

\begin{abstract}
The final equilibrium state and the spreading dynamics approaching equilibrium have been studied for a sessile drop posed on a thin solid. For the case of equilibrium, both axisymmetric and cylindrically symmetrical geometries have been considered. In both situations, but particularly the latter, liquid surface tension can markedly curve the initially planar solid. The dynamics of spreading can be speeded up by the presence of a thin substrate. Released elastic energy contributes to capillary effects in overcoming viscous resistance to flow.
\end{abstract}

\section{Introduction.}

Interest in the configuration of liquid surfaces in contact with solids has been shown for nearly two centuries. The two classic contributions are due to Laplace [1] and to Young [2] ; the former elucidating the equation giving the equilibrium shape of the liquid/surrounding fluid interface and the latter giving the relationship between the three surface/interfacial tensions, $\gamma_{i j}$, and the equilibrium contact angle, $\Theta$, at the triple line liquid/fluid/solid (although in fact, the mathematical formulation of this was never given in the much cited reference [2]).

Both Laplace and Young made use of force arguments but it can be shown, using variational arguments, that the general conclusions are correct (e.g. [3-7]), despite criticisms put forward at various times [8-10]. By treating the $\gamma_{i j}$ as free energy terms, a minimum of the total free energy of the system is sought. Force and energy considerations lead to the same conclusions and, provided the solid is perfectly rigid and the contact angle is not too small (otherwise a non-negligible influence of van der Waals forces may be felt-see, for example, references [11-13]), these results are valid for the majority of experimental cases.

Capillary forces may be regarded as small and the aforementioned assumption of solid rigidity is thus a very good approximation for macroscopic situations concerning high modulus solids. Any slight strain in the solid is negligible. However, if the solid is relatively soft and/or very thin, such as can be found in organic contexts (cell walls, membranes), then solid deformation cannot necessarily be ignored. Significant strain of the solid caused by capillary effects may markedly influence the overall behaviour of the liquid/fluid/solid system. In the case of a soft bulk material, a wetting ridge may form at the triple line [14] and under certain conditions, this may influence contact angle equilibrium [15]. For a substrate consisting of an initially planar thin solid, membrane or plate, an important degree of curvature may result $[16,17]$. In the present paper, we shall consider the latter case, that of a thin elastic plate. Initially we shall investigate the overall conformation of a liquid drop on such a solid. However, the interest of such a system is potentially its applicability to living biological situations when the 
dynamics of evolution towards the equilibrium state is fundamental. Although the comprehension of spreading and rolling dynamics is still in its infancy relatively speaking $[13,18]$, we shall attempt to understand the evolution towards equilibrium, or spreading dynamics, when a liquid drop is placed on a thin elastic plate.

\section{Axisymmetric equilibrium configuration.}

In figure 1 is represented the side view of an axisymmetric sessile drop of radius $r_{\mathrm{e}}$ of liquid, L, resting on a disc of radius $a$ of solid, $S$, in the presence of the surrounding fluid, which we shall suppose is vapour, $\mathrm{V}$. The solid disc, to be modelled by thin plate theory, is planar before deposition of the liquid drop and the curvature shown schematically is due to an attempt by the system to minimise

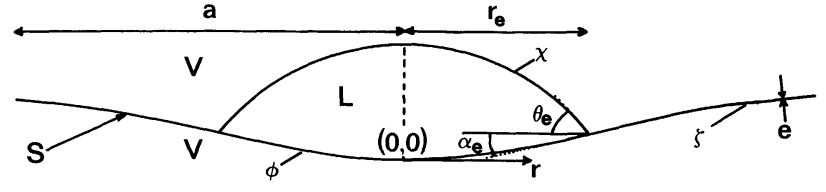

Fig. 1. - Side view of axisymmetric drop on thin disc.

its surface (interfacial) free energy whilst having the elastic constraint of strain energy associated with the solid deformation. There is an interfacial free energy, $\gamma_{i j}$, applicable to each contact zone where $i$ and $j$ may be L, S or V. Gravitational energy will here be neglected (small drops or similar $\mathrm{L}$ and $\mathrm{V}$ densities).

For axisymmetry, we may thus write the overall free energy of the system, $E_{\mathrm{T}}$ :

$E_{\mathrm{T}} / 2 \pi=\int_{0}^{r_{\mathrm{e}}}\left[\left(\gamma_{\mathrm{SL}}+\gamma_{\mathrm{SV}}\right) r\left(1+\phi_{r}^{2}\right)^{1 / 2}+\gamma r\left(1+\chi_{r}^{2}\right)^{1 / 2}+r E_{\mathrm{E}}\right] \mathrm{d} r+\int_{r_{\mathrm{e}}}^{a}\left[2 \gamma_{\mathrm{SV}} r\left(1+\zeta_{r}^{2}\right)^{1 / 2}+r E_{\mathrm{E}}\right] \mathrm{d} r$,

where $E_{\mathrm{E}}$ represents the strain energy density [19] :

$$
\begin{aligned}
E_{\mathrm{E}}=\frac{D}{2}\left[\frac{\phi_{r r}^{2}}{\left(1+\phi_{r}^{2}\right)^{5 / 2}}+\frac{\phi_{r}^{2}}{r^{2}\left(1+\phi_{r}^{2}\right)^{1 / 2}}\right. & \\
& \left.+\frac{2 \nu \phi_{r} \phi_{r r}}{r\left(1+\phi_{r}^{2}\right)^{3 / 2}}\right] .
\end{aligned}
$$

Equation (2) is valid for $r \leqslant r_{\mathrm{e}}$. A similar expression with $\zeta$ replacing $\phi$ applies to $r \geqslant r_{\mathrm{e}}$. In the above expressions, $\gamma=\gamma_{\mathrm{LV}}, D$ and $\nu$ are respectively the flexural rigidity and Poisson's ratio of the plate [ $D=E e^{3} / 12\left(1-\nu^{2}\right)$ where $E$ is Young's modulus and $e$ is the plate thickness]. The two solid profiles denoted $\phi$ and $\zeta$, and that of the liquid/vapour interface, $\chi$, are functions of the cylindrical coordinate $r$ as shown in figure 1 .

At equilibrium, $E_{\mathrm{T}}$ must be a minimum with the constraint of constant drop volume, $V$ :

$$
V / 2 \pi=\int_{0}^{r_{\mathrm{e}}} r(\chi-\phi) \mathrm{d} r .
$$

This amounts to a variational problem in which we minimise the function $J\left[=\left(E_{\mathrm{T}}-\Delta p \cdot V\right) / 2 \pi\right.$ where $\Delta p$ is a Lagrange multiplier and equals the Laplacian liquid/vapour pressure difference]. The variational treatment is excluded here since it is somewhat lengthy, but the essential results may be found in reference [16]. Three identities concerning equilibrium at the triple line and a differential equation describing each profile, $\phi, \zeta$ and $\chi$, are obtained. After some algebra, the identities reduce to two expressions :

$$
\gamma_{\mathrm{SV}}=\gamma_{\mathrm{SL}}+\gamma \cos \left(\theta_{\mathrm{e}}+\alpha_{\mathrm{e}}\right)
$$

$\gamma \sin \left(\theta_{\mathrm{e}}+\alpha_{\mathrm{e}}\right)=D \cos \alpha_{\mathrm{e}}$

$$
\times\left[\left.\frac{\mathrm{d}}{\mathrm{d} r}\left(\frac{1}{R^{(2)}}\right)\right|_{r_{\mathrm{e}}}-\left.\frac{\mathrm{d}}{\mathrm{d} r}\left(\frac{1}{R^{(1)}}\right)\right|_{r_{\mathrm{e}}}\right]
$$

where $\theta_{\mathrm{e}}$ and $\alpha_{\mathrm{e}}$ represent the equilibrium angles shown in figure 1 , and $R^{(1)}$ and $R^{(2)}$ represent the radii of curvature of the solid at $r_{\mathrm{e}}$ when approaching respectively from inside and from outside the drop. Equation (4) amounts to Young's equation since the equilibrium contact angle is given by $\Theta=\left(\theta_{\mathrm{e}}+\alpha_{\mathrm{e}}\right)$. Equation (5) can in a sense be considered as representing the force balance perpendicular to the plate at $r_{\mathrm{e}}$ where the component of surface tension $\gamma$ is balanced by an elastic deformation term in the solid involving the flexural rigidity and local curvature. Nevertheless, a full appraisal of this aspect should involve a discussion of the wetting ridge described elsewhere [14, 15].

Of the three differential equations obtained, that for the liquid/vapour interface, $\chi$, is simply the standard axisymmetric capillary equation which, in the absence of gravity, has the simple solution of a circular arc. Both solid profile equations turn out to be rather complex involving the 4th derivatives of $\phi$ and $\zeta$ with respect to $r$. Nevertheless certain simplifying assumptions may be made and it is found that the solid profile may be approximated by a circular arc under the drop and a logarithmic function outside [16]. We may thus write :

$$
\begin{gathered}
\chi \sim\left(P^{2}-r^{2}\right)^{1 / 2}+R-\left(P^{2}-r_{\mathrm{e}}^{2}\right)^{1 / 2}-\left(R^{2}-r_{\mathrm{e}}^{2}\right)^{1 / 2}, \\
\phi \sim R-\left(R^{2}-r^{2}\right)^{1 / 2}, \\
\zeta \sim r_{\mathrm{e}}^{2}\left(R^{2}-r_{\mathrm{e}}^{2}\right)^{-1 / 2} \cdot \ln r+\text { Cst. },
\end{gathered}
$$


where $R$ and $P$ are the lower and upper drop surface curvatures respectively. These equations have been employed to study the effect of plate rigidity on the overall configuration of the liquid/vapour/solid system. The approximations of $a \gg r_{\mathrm{e}}$ and $R \gg r_{\mathrm{e}}$ are made and an expression for $J$ may be obtained. This expression may be treated by standard methods of the differential calculus. Setting the three differentials of $J$ with respect to $P, R$ and $r_{\mathrm{e}}$ to zero and using the constancy of drop volume, a system of equations is obtained. Allowing $r_{\mathrm{e}}$ to remain constant and assuming $\alpha_{\mathrm{e}}$ relatively small such that $\sin \alpha_{\mathrm{e}} \sim$ $\alpha_{\mathrm{e}}$, we may solve to obtain an approximate expression for $\alpha_{\mathrm{e}}$ (a quadratic equation in $\alpha_{\mathrm{e}}^{2}$ ). This expression has been used to consider the variation of $\alpha_{\mathrm{e}}$ with plate thickness. (Basic details may be found in reference [17]).

Figure 2 gives two examples of $\alpha_{\mathrm{e}}$ versus $e$, plate thickness. The angle $\theta_{\mathrm{e}}$ may be obtained simply by difference given that $\left(\theta_{\mathrm{e}}+\alpha_{\mathrm{e}}\right)=\Theta$ is a constant for a given set of surface/interfacial tensions (cf. Eq. (4)).

In the examples chosen, we have taken $\gamma_{\mathrm{SL}}=$ $\gamma_{\mathrm{SV}}=30 \mathrm{~mJ} \cdot \mathrm{m}^{-2} \quad\left(\Theta=90^{\circ}\right), \quad E=10^{8} \quad \mathrm{~N} \cdot \mathrm{m}^{-2}$, $\nu=1 / 3$ and $r_{\mathrm{e}}=10^{-3} \mathrm{~m}$. Curves shown correspond to $\gamma=10$ and $\gamma=20 \mathrm{~mJ} \cdot \mathrm{m}^{-2}$. At very low values of $e$, the approximations used are likely to fail ; a cutoff is therefore shown below about $5 \mu \mathrm{m}$. It can be seen that for $e$ less than about $20 \mu \mathrm{m}$, the curvature of the plate can become quite marked with $\alpha_{\mathrm{e}}$ being over $1^{\circ}$ and increasing with decreasing plate thickness to the order of $10^{\circ}$ or more. $\theta_{\mathrm{e}}$ shows a corresponding drop. However, above a value of about $30 \mu \mathrm{m}$ for $e$, plate deformation becomes feeble and $\alpha_{\mathrm{e}}$ tends asymptotically to zero as would be expected on a rigid solid. It can thus be seen that whilst the intrinsic contact angle of a drop placed on a thin plate is constant (ignoring effects of the wetting ridge mentioned earlier) and governed by Young's equation (4), the apparent contact angle as seen from the side and with respect to the horizontal can be significantly reduced by effects of solid curvature. As may be seen in figure 2, this curvature is directly related to liquid surface tension. Although the approximate analysis adopted here would be insufficient for an accurate assessment, the effects of a drop of a very high surface tension liquid (such as mercury) on thin plate curvature could be very significant (provided, of course, that $\Theta$ is not too close to zero or $\left.180^{\circ}\right)$.

\section{Cylindrical equilibrium.}

In a previous paper [17] it was shown that although in most situations the axisymmetric equilibrium state described above will prevail, there may well be cases in which departures from this configuration occur. Three-dimensional problems are also generally more complex to handle than those in two dimensions. For these reasons, we shall consider the dynamics of spreading of a liquid drop on a thin solid in its twodimensional context, i.e. that corresponding to the vertical section of a long cylindrical drop. However, before describing the dynamics, let us consider the

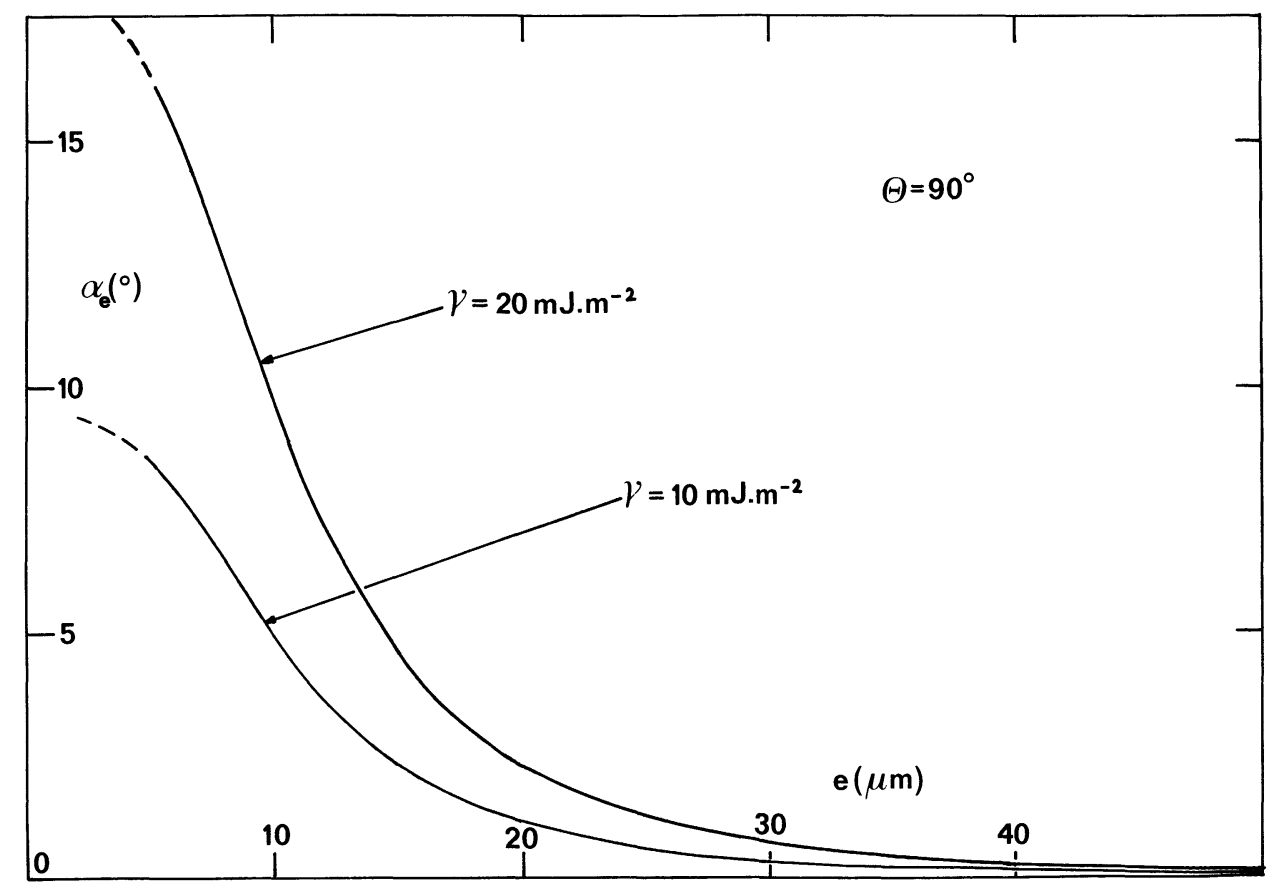

Fig. 2. - Plate inclination angle, $\alpha_{\mathrm{e}}$, vs. plate thickness, $e$, for $\gamma=10$ and $20 \mathrm{~mJ} . \mathrm{m}^{-1}$ (see text for details). 
equilibrium statics of the system and compare with the axisymmetric case. The situation is considerably simpler to treat in two dimensions and simple force analysis may be employed rather than resorting to variational calculus and energy minimisation.

Consider figure 3 which represents one half of a vertical section taken through a cylindrical drop, we may treat just one half because of symmetry considerations. There exists an excess (Laplace) pressure

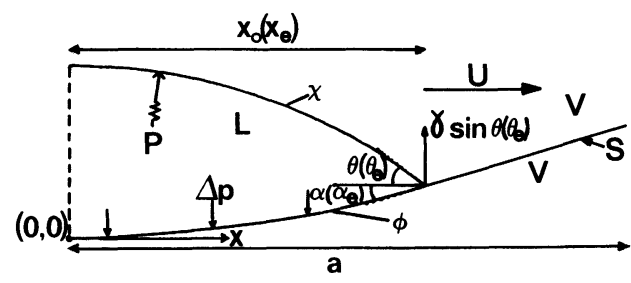

Fig. 3. - Side view of half cylindrical drop.

within the drop which results form the liquid surface tension and the curvature of the liquid/vapour interface. Ignoring gravity, this pressure is everywhere constant, $\Delta p$, and for equilibrium of the thin plate, $\Delta p$ acting downwards over the half drop width $x_{\mathrm{e}}$ will be balanced by $\gamma \sin \theta_{\mathrm{e}}$ acting upwards (per unit length of triple line). We can thus evaluate $\Delta p=\gamma \sin \theta_{\mathrm{e}} / x_{\mathrm{e}}$. Although the forces balance, there will be a net bending moment, $M$, tending to curve the solid. At a given value of $x$ this is given by :

$$
\begin{aligned}
& M(x)=\gamma\left(x_{\mathrm{e}}-x\right) \sin \theta \\
&-\Delta p \int_{x}^{x_{\mathrm{e}}}(t-x) \mathrm{d} t=\frac{\gamma \sin \theta}{2}\left(x_{\mathrm{e}}-\frac{x^{2}}{x_{\mathrm{e}}}\right),
\end{aligned}
$$

where $x$ is the distance from the drop centre and $t$ represents $x$ before integration. If we assume that the degree of bending of the plate is relatively small $\left(\phi_{x} \ll 1\right)$, we may apply simple plate (beam) theory (20) :

$$
M=D \phi_{x x} .
$$

Simple integration and use of the facts that $\phi(0)=$ $\phi_{x}(0)=0$ lead to :

$$
\phi(x)=\frac{\gamma \sin \theta_{\mathrm{e}}}{4 D}\left(x_{\mathrm{e}} x^{2}-\frac{x^{4}}{6 x_{\mathrm{e}}}\right) .
$$

Assuming angle $\theta_{\mathrm{e}}$ to be fairly small :

$$
\alpha_{\mathrm{e}} \sim \tan \alpha_{\mathrm{e}}=\phi_{x}\left(x_{\mathrm{e}}\right) \sim x_{\mathrm{e}}^{2} \gamma \theta_{\mathrm{e}} / 3 D,
$$

and using the constancy of $\Theta=\left(\theta_{\mathrm{e}}+\alpha_{\mathrm{e}}\right)$ and definition of $D$ :

$$
\alpha_{\mathrm{e}} \sim \frac{4 \gamma\left(1-\nu^{2}\right) x_{\mathrm{e}}^{2} \Theta}{E e^{3}+4 \gamma\left(1-\nu^{2}\right) x_{\mathrm{e}}^{2}} .
$$

Equation (13) has been used to get an idea of the variation of $\alpha_{\mathrm{e}}$ with plate thickness, $e$. Figure 4 represents calculated results for a hypothetical system in which $\Theta=30^{\circ}, \quad \gamma=30 \mathrm{~mJ} . \mathrm{m}^{-2}, \quad \gamma_{\mathrm{SL}}=$ $20 \mathrm{~mJ} \cdot \mathrm{m}^{-2}, \gamma_{\mathrm{SV}}=46 \mathrm{~mJ} \cdot \mathrm{m}^{-2}$ (this last value being imposed by the value of $\Theta), E=10^{8} \mathrm{~N} \cdot \mathrm{m}^{-2}$, $\nu=1 / 3$ and $x_{\mathrm{e}}=10^{-3} \mathrm{~m}$.

The corresponding curve for the three-dimensional case having the same characteristics and calculated as discussed in the last section $\left(x_{\mathrm{e}}=r_{\mathrm{e}}\right)$ is also given. In both cases, a cut-off has been given at about $5 \mu \mathrm{m}$ since below this thickness, the calculated values of $\alpha_{\mathrm{e}}$ become sufficiently large for the initial assumptions of the simple analyses to become doubtful. It can be seen that in both cases, the inclination of the

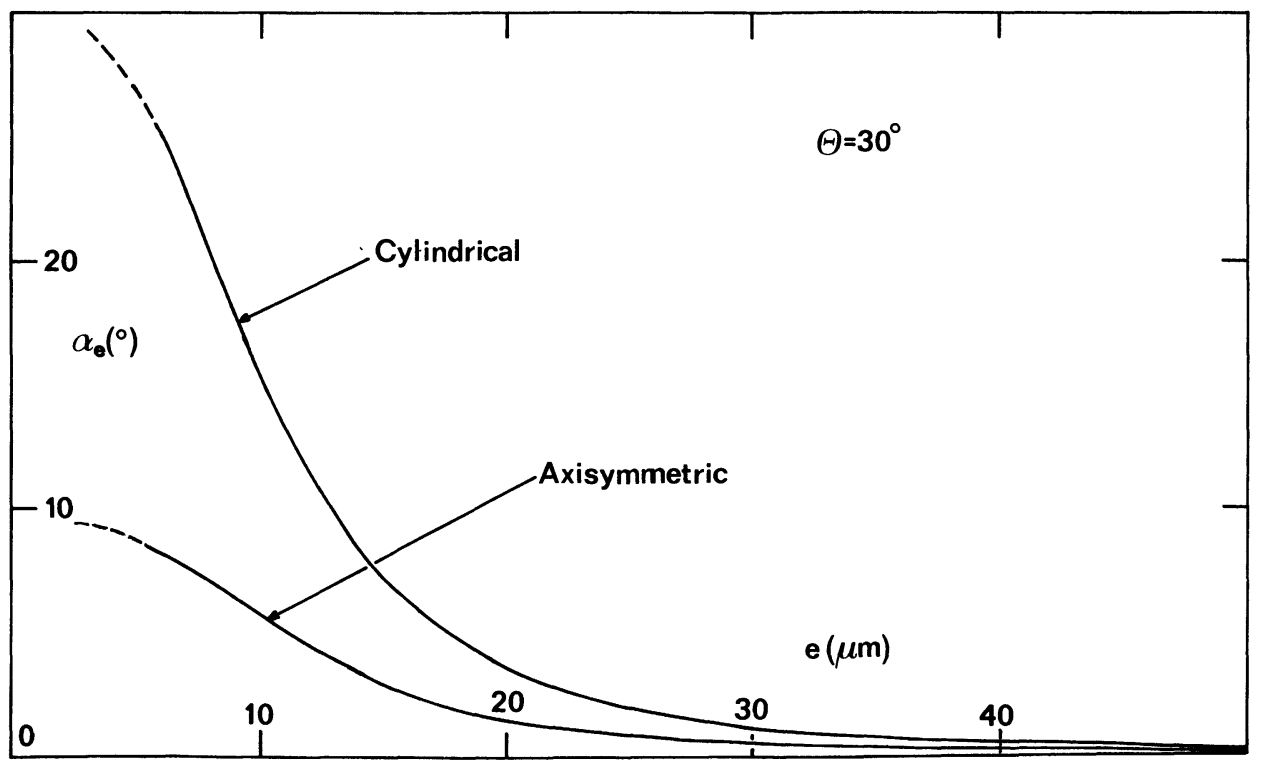

Fig. 4. - Angle $\alpha_{\mathrm{e}} v s$. $e$ for cylindrical and axisymmetric drops (see text for details). 
thin plate at the triple line becomes quite marked for low values of e but that in particular this is true for the cylindrical drop. This is a consequence of the bending mode. Cylindrical bending is relatively easy to effect whereas spherical bending invokes side effects since two orthogonal bending directions (and radii of curvature) are involved (cf. Eq. (2)). In addition, the exterior part of the plate has an effect in the axisymmetric case which is absent for the cylindrical geometry.

\section{Spreading dynamics.}

Having established that the wetting equilibrium of a cylindrical drop on a thin plate can involve an important degree of solid curvature, let us consider the evolution of such a system after the initial pose of a drop of liquid until the final equilibrium is attained. In general the initial contact angle $(\theta+\alpha)$, will be greater than its equilibrium value, $\Theta$, and the half drop width, $x_{0}$, will be less than $x_{\mathrm{e}}$. Nevertheless, vertical (in the sense of Fig. 3) equilibrium for a given value of $x_{0}$ will be rapidly achieved (we assume, at least) and the rate-determining step in the evolution towards final, overall equilibrium will be the horizontal spreading and growth of $x_{0}$. In this case, equation (11) will describe the plate profile (with $x_{0}$ replacing $x_{\mathrm{e}}$ and $\theta$ replacing $\left.\theta_{\mathrm{e}}\right)$ and the liquid/vapour interface will be circular :

$$
\begin{aligned}
\chi(x)=\left(P^{2}-x^{2}\right)^{1 / 2}-\left(P^{2}-\right. & \left.x_{0}^{2}\right)^{1 / 2} \\
& +5 \gamma x_{0}^{4} / 24 D P .
\end{aligned}
$$

The volume of the drop (per unit length of triple line) is a constant :

$$
V=\int_{0}^{x_{0}}(\chi-\phi) \mathrm{d} x \sim \frac{x_{0}^{2} \theta}{3}\left(1+\frac{2 \gamma x_{0}^{2}}{5 D}\right) .
$$

As before we assume here $\theta$ small and $\alpha$ very small.

The free energy of the system may then be expressed :

$$
\begin{aligned}
E_{\mathrm{T}} \sim \int_{0}^{x_{0}}\left[\gamma\left(1+\frac{\chi_{x}^{2}}{2}\right)\right. & +\left(\gamma_{\mathrm{SL}}-\gamma_{\mathrm{SV}}\right) \\
& \left.+\frac{D}{2} \phi_{x x}^{2}\right] \mathrm{d} x+2 \gamma_{\mathrm{SV}} a
\end{aligned}
$$

Equation (16) can be compared to equations (1) and (2). It is simpler since we assume $\phi_{x} \ll 1$ and the elastic term involves only one radius of curvature under the drop. The plate remains undeformed outside. Evaluation of equation (16) will be done for two limiting cases. In the first, we assume $\Theta$ finite and $D$ large such that we may neglect terms of $O\left(D^{-2}\right)$.

In the second case we shall be interested in $\Theta$ zero and therefore consider $x_{0}$ large since $x_{\mathrm{e}} \rightarrow \infty$.

REVUE DE PHYSIQUE APPLIQUÉE. - T. 23, $\mathrm{N}^{\circ} 6$, JUIN 1988
1. FINITE EQUILIBRIUM CONTACT ANGLE. - Taking initially the case of large $D$, equations $(11,14,15$, 16) lead to :

$$
\begin{aligned}
E_{\mathrm{T}} \sim\left(\gamma+\gamma_{\mathrm{SL}}-\gamma_{\mathrm{SV}}\right) & x_{0}+\frac{3 \gamma V^{2}}{x_{0}}\left[\frac{1}{2 x_{0}^{2}}-\frac{\gamma}{5 D}\right] \\
+ & 2 \gamma_{\mathrm{SV}} a+O\left(D^{-2}\right) .
\end{aligned}
$$

During spreading, $E_{\mathrm{T}}$ will decrease towards equilibrium at a rate given by $U . \mathrm{d} E_{\mathrm{T}} / \mathrm{d} x_{0}$ where $U$ is the advancing speed of the triple line, $\mathrm{d} x_{0} / \mathrm{d} t$,

$$
\begin{aligned}
U \cdot \frac{\mathrm{d} E_{\mathrm{T}}}{\mathrm{d} x_{0}} \sim\left(\gamma+\gamma_{\mathrm{SL}}\right. & \left.-\gamma_{\mathrm{SV}}\right) \\
& \times U-\frac{3 \gamma V^{2}}{x_{0}^{2}}\left[\frac{3}{2 x_{0}^{2}}-\frac{\gamma}{5 D}\right] \\
& \times U \sim \frac{\gamma}{2}\left[\Theta^{2}-(\theta+\alpha)^{2}\right] \cdot U .
\end{aligned}
$$

This decrease in $E_{\mathrm{T}}$ will be consumed during spreading, essentially as dissipation due to liquid viscosity, $\eta$. Viscous dissipation has already been studied for the case of spreading on a rigid substrate $[13,21]$ and we shall use the results quoted, but modifying for the fact that in the present case, the effective contact angle is $(\theta+\alpha)$ :

$$
T \stackrel{\circ}{\sim} \frac{3 \eta U^{2} l}{(\theta+\alpha)} .
$$

The dissipation (per unit length of triple line), $T \stackrel{\circ}{S}$, depends on $l=\ln \left[\frac{x_{0}-x_{\min }}{x_{0}-x_{\max }}\right]$ where $x_{\min }$ and $x_{\max }$ are respectively near the origin and the triple line, although their exact positions are here undefined. Since $l$ is a logarithmic function, little error will be introduced considering it as constant.

Since $T \stackrel{\circ}{S}+U . \mathrm{d} E_{\mathrm{T}} / \mathrm{d} x_{0} \sim 0$, we obtain from equations $(12,15,18,19)$, a differential equation for the spreading speed, $\mathrm{d} x_{0} / \mathrm{d} t$ :

$$
\begin{aligned}
\frac{\mathrm{d} x_{0}}{\mathrm{~d} t} \sim \frac{3 V^{3} V^{*}}{2 l} & {\left[\frac{3}{x_{0}^{6}}-\frac{3 \gamma}{5 D x_{0}^{4}}\right.} \\
& \left.-\frac{1}{x_{0}^{2}}\left(\frac{3}{x_{\mathrm{e}}^{4}}-\frac{2 \gamma}{5 D x_{\mathrm{e}}^{2}}\right)+\frac{\gamma}{5 D x_{\mathrm{e}}^{4}}\right],
\end{aligned}
$$

where $V^{*}$ is the characteristic capillary speed $(=\gamma / \eta)$.

Solving equation (20) for $x_{0} \rightarrow x_{\mathrm{e}}$, we find approximately exponential behaviour (Ref. [21]) such that the time constant, $\tau$ is given by :

$$
\tau \sim \frac{3 l x_{\mathrm{e}}}{2 V^{*} \theta_{\mathrm{e}}^{3}} \cdot\left(1+\frac{16 \gamma x_{\mathrm{e}}^{2}}{15 D}\right)^{-1} .
$$

Taking $\quad \gamma \sim 30 \mathrm{~mJ} . \mathrm{m}^{-2}, \quad x_{\mathrm{e}} \sim 10^{-3} \mathrm{~m} \quad$ and $D \sim 10^{-7} \mathrm{Nm}$ (corresponding to a solid of $E \sim 10^{8} \mathrm{Nm}^{-2}, e \sim 20 \mu \mathrm{m}$, for example), we find the 
time constant decreased by about $30 \%$ and correspondingly the relative dynamics towards final equilibrium is that much faster than on a rigid solid. (N.B. absolute comparison of dynamics between flexible and rigid cases is of little use since the final equilibrium states are different.)

Equation (21) may be applied to the case of an elastomer. Strictly speaking, in this case the flexural rigidity, $D$, should involve the complex modulus, $E^{*}$, of the solid and thus energy dissipation due to mechanical hysteresis would appear in equation (20) (a separate study considers this effect in the case of the wetting ridge [22]). Nevertheless we assume here that spreading dynamics is sufficiently rapid for the loss component to be negligible. In this case, the elastic modulus of the material is given by:

$$
E=m k T / N v
$$

where $N$ equals the number of monomers of volume $v$ between crosslinks, $k T$ represents the usual Boltzmann constant times absolute temperature and $m$ is a numerical factor, about 1 [23]. The diameter of an elastomeric monomer, $d$, is given approximately by $\gamma v / k T$ and thus, provided the plate thickness, $e$, is not too feeble, the time constant, $\tau$, for the exponential approach to equilibrium wetting may be written :

$$
\tau \sim l e^{3} / 6 V^{*} \theta_{\mathrm{e}}^{3} x_{\mathrm{e}} N d,
$$

and we see that the spreading dynamics is directly related to the degree of crosslinking of the elastomer.

2. ZERO EQUILIBRIUM CONTACT ANGLE. - For the case of $\Theta$ zero, we consider the final stages of spreading, i.e. taking $\gamma x_{0}^{2} / D$ to be large. Equation (16) then becomes :

$$
\begin{aligned}
E_{\mathrm{T}} \sim(\gamma+ & \left.\gamma_{\mathrm{SL}}-\gamma_{\mathrm{SV}}\right) x_{0}+\frac{15 D V^{2}}{4 x_{0}^{5}} \\
& -\frac{75 D^{2} V^{2}}{8 \gamma x_{0}^{7}}-\frac{375 D^{3} V^{2}}{8 \gamma^{2} x_{0}^{9}}+2 \gamma_{\mathrm{SV}} a
\end{aligned}
$$

Using the same procedure of equating rate of loss of $E_{\mathrm{T}}$ to dissipative viscous flow leads to the following differential equation :

$$
\frac{\mathrm{d} x_{0}}{\mathrm{~d} t} \sim \frac{5 V}{6 \eta l x_{0}^{2}}\left[\frac{75 D V^{2}}{4 x_{0}^{6}}+\Gamma\right]+O\left(x_{0}^{-10}\right),
$$

where $\Gamma=\left(\gamma_{\mathrm{SV}}-\gamma_{\mathrm{SL}}-\gamma\right)$.

Now $\Gamma$ will be positive or zero. If it is zero, Antonov's equality is satisfied. In this case, which seems to be usual [24], equation (25) gives :

$$
x_{0}^{9} \sim \frac{1125 V^{3} D t}{8 \eta l}
$$

The scaling law thus shows an increase in spreading distance $x_{0}$ which depends on $t^{1 / 9}$. What,.however, is more interesting is that the spreading is virtually independent of the value of $\gamma$, since this only occurs in lower order terms. At long times approaching spreading equilibrium, the rate of increase of $x_{0}$ is essentially conditioned by competition between elastic effects favouring wetting and viscous dissipation braking the phenomenon.

If we assume $\Gamma$ to be positive, the term in $x_{0}^{-6}$ can be considered (relatively) negligible and we obtain :

$$
x_{0}^{3} \sim 5 V t \Gamma / 2 \eta l .
$$

Distance $x_{0}$ then depends on $t^{1 / 3}$. Competition here exists between the spreading tendancy characterised by $\Gamma$ and the viscous slowing down. The solid has become sufficiently planar for elastic effects to become unimportant.

\section{Conclusions.}

Wetting phenomena are important in many branches of science and technology. In the majority of cases treated on a macroscopic scale, to a very good degree of approximation, a solid phase in contact with a liquid may be considered to be rigid. However in certain microscopic contexts, especially those involving biological systems, the assumption of rigidity will leave something to be desired. Such things as cell walls are quite flexible and prone to deformation by capillary forces. We have therefore considered aspects of wetting in which solid strain is provoked by interfacial tensions. The solid has been modelled, albeit somewhat idealistically, by thin plate theory. Static, equilibrium configurations have been analysed using both variational techniques and simple mechanical arguments. An initially planar thin solid may become appreciably curved by the presence of a liquid drop, particularly in a system showing cylindrical symmetry. Static equilibrium is obtained after spreading. It is shown that the dynamics of spreading may be speeded up on a thin solid by strain effects. Elastic energy released may contribute to capillary effects in order to overcome viscous resistance to flow. Under certain conditions, the final stages of approach to wetting equilibrium are controlled essentially by solid elasticity and liquid viscosity, the actual liquid surface tension playing a minor role. 


\section{References}

[1] Laplace, P. S., Mécanique Céleste, Suppl. au $X^{\mathrm{e}}$ livre (Coureier, Paris) 1805.

[2] Young, T., Phil. Trans. R. Soc. London 95 (1805) 65.

[3] Johnson, R. E., J. Phys. Chem. 63 (1959) 1655.

[4] Collins, R. E., Cooke, C. E., Trans. Faraday Soc. 55 (1959) 1602.

[5] Goodrich, F. C., Surface and Colloid Science, Ed. E. Matijević (Wiley, New York) 1, 1969, chapitre 1.

[6] Fortes, M. A., Phys. Chem. Liq. 9 (1980) 285.

[7] Shanahan, M. E. R., Adhesion 6, Ed. K. W. Allen (Appl. Sci. Pub., London) 1982, chapitre 5.

[8] Bikerman, J. J., J. Phys. Chem. 63 (1959) 1658.

[9] Idem, Physical Surfaces (Academic Press, London) 1970, chapitre 6.

[10] PethicA, B. A., Pethica, T. J. P., Proc. Intern. Congr. Surf. Activity (2nd, London) 3 (1957) 131.

[11] Johnny, J. F., De Gennes, P. G., C. R. Acad. Sci. Paris 299 II (1984) 279.
[12] Idem, ibid. 303 II (1986) 337.

[13] de Gennes, P. G., Rev. Mod. Phys. 57 (1985) 827.

[14] Shanahan, M. E. R., DE Gennes, P. G., Adhes. 11, Ed. K. W. Allen (Elsevier Appl. Sci. Pub., London) 1987, chapitre 5.

[15] Shanahan, M. E. R., J. Phys. D 20 (1987) 945.

[16] Idem, J. Adhes. 18 (1985) 247.

[17] Idem, ibid. 20 (1987) 261.

[18] Dussan V, E. B., Ann. Rev. Fluid. Mech. 11 (1979) 371.

[19] Timoshenko, S. P., Goodier, J. N., Theory of Elasticity, 3rd Ed. (Mc Graw-Hill, New York) 1970 , p. 246.

[20] Idem, ibid., p. 288.

[21] De Gennes, P. G., C. R. Acad. Sci. Paris 298 II (1984) 111.

[22] Shanahan, M. E. R., ibid. 306 II (1988) 113.

[23] Treloar, L. R. G., The Physics of Rubber Elasticity (Clarendon, Oxford) 1949, p. 66.

[24] Cahn, J. W., J. Chem. Phys. 66 (1977) 3667. 